\title{
TARC/CCL17 gene polymorphisms and expression associated with susceptibility and coronary artery aneurysm formation in Kawasaki disease
}

\author{
Chiu-Ping Lee', Ying-Hsien Huang ${ }^{1}$, Yu-Wen Hsu' ${ }^{2}$, Kuender D. Yang ${ }^{3}$, Hsu-Chen Chien², Hong-Ren Yu' ${ }^{1}$ Ya-Ling Yang ${ }^{4}$, \\ Chih-Lu Wang ${ }^{5}$, Wei-Chiao Chang ${ }^{2}$ and Ho-Chang Kuo ${ }^{1}$
}

BACKGROUND: Kawasaki disease (KD) is a systemic vasculitis of unknown etiology. Thymus and activation-regulated chemokine/chemokine ligand 17 (TARC/CCL17) is one of the Th2 chemokines and has been suggested as a candidate gene for conferring susceptibility to Th2 associated with allergy diseases. This study examined the correlation between gene polymorphisms and plasma levels of TARC/CCL17 in patients with $\mathrm{KD}$ and the outcomes of KD.

METHODS: A total of $381 \mathrm{KD}$ patients and 564 controls were subjected to determination of five tagging single-nucleotide polymorphisms of TARC/CCL17. In addition, plasma TARC/ CCL17 levels were measured by enzyme-linked immunosorbent assay.

RESULTS: Polymorphisms of TARC/CCL17 were significantly different between normal children and patients with KD. A allele of rs4784805 has better intravenous immunoglobulin (IVIG) treatment response to KD. Furthermore, plasma TARC/ CCL17 levels were higher in KD patients than that in controls before IVIG treatment. After IVIG treatment, plasma TARC/ CCL17 levels decreased significantly.

CONCLUSION: This study provides the first evidence supporting the association between TARC/CCL17 polymorphisms, susceptibility of KD, and IVIG responses in KD patients.

K awasaki disease (KD), also known as mucocutaneous lymph node syndrome, is an acute febrile systemic vasculitis that was first described by Kawasaki et al. in 1974 in English (1), but its etiology remains unknown till now. It occurs most commonly in children younger than $5 \mathrm{y}$ of age, particularly in children younger than $2 \mathrm{y}$ of age, and the clinical presentation of $\mathrm{KD}$ is prolonged fever, conjunctivitis, diffuse mucosal inflammation, polymorphous skin rashes, indurative edema of the hands and feet associated with peeling of finger tips, and nonsuppurative lymphadenopathy (2). In developed countries, KD has been the leading cause of acquired heart diseases in children $(2,3)$. The most serious complication of $\mathrm{KD}$ is the occurrence of coronary artery lesions (CALs), including myocardial infarction, coronary artery fistula formation (4), coronary artery dilation, or coronary artery aneurysm (CAA) (5). In addition, $\mathrm{KD}$ is the major cause of acquired CAA in childhood (6). The prevalence of KD in children is the highest in Japan, followed by Korea and Taiwan, and the lowest in Europe (5). Therefore, it is possible that a genetic background plays an important role in the pathogenesis of KD.

Thymus and activation-regulated chemokine/chemokine ligand 17 (TARC/CCL17) is a member of the CC chemokine group (7). It is a ligand of the CC chemokine receptor (8), which is selectively expressed on Th2 cells (9) and serves for the recruitment and migration of cells bearing this receptor (7-9). TARC/CCL17 has been suggested as a candidate gene for conferring susceptibility to Th2 associated with allergy diseases and is highly implicated in the pathogenesis of atopic dermatitis $(10,11)$ and bronchial asthma (12). There are several lines of evidence pointing out an abnormal Th1/Th2 balance in $\mathrm{KD}$ patients (13). Recently, it has been shown that the incidence of atopic dermatitis and bronchial asthma among children with $\mathrm{KD}$ was nine times and three times greater than that of controls $(14,15)$, respectively. In addition, a single-nucleotide polymorphism (SNP) in a C-to-T substitution at position $-431(431 \mathrm{C} / \mathrm{T})$ in the $5^{\prime}$-flanking region was demonstrated that significantly increased the levels of serum TARC/CCL17 concentrations (16). Therefore, this study examined the correlation between TARC/CCL17 gene polymorphisms and plasma levels of TARC/CCL17 in patients with KD and the outcomes of $\mathrm{KD}$.

\section{RESULTS}

Association of $T A R C / C C L 17$ Polymorphism With Susceptibility to KD

Table 1 shows the characteristics of the subjects. A total of 381 $\mathrm{KD}$ patients and 564 controls were enrolled in this study. In the selected population, $66.8 \%$ of cases and $55.5 \%$ of controls were male. The mean age of patients and controls was $1.7 \pm 1.6$ $y(S D)$ and $5.4 \pm 3.7 y$, respectively. Children were predominant

\footnotetext{
The first three authors contributed equally to this work

'Department of Pediatrics, Kaohsiung Chang Gung Memorial Hospital and Chang Gung University College of Medicine, Kaohsiung, Taiwan; ${ }^{2}$ Department of Clinical Pharmacy, College of Pharmacy, Taipei Medical University, Taipei, Taiwan; ${ }^{3}$ Department of Medical Research and Pediatrics, Show Chwan Memorial Hospital in Chang Bing, Changhua, Taiwan; ${ }^{4}$ Department of Anesthesiology, Kaohsiung Chang Gung Memorial Hospital and Chang Gung University College of Medicine, Kaohsiung, Taiwan; ${ }^{5}$ Po-Jen Hospital, Kaohsiung, Taiwan. Correspondence: Ho-Chang Kuo (erickuo48@yahoo.com.tw)
} 
in the study population. There were $9.7 \%$ of KD with CAL formation and $4.2 \%$ with aneurysm formation. Among these KD patients, $12.9 \%$ were resistant to initial intravenous immunoglobulin (IVIG) treatment. The distribution of SNP genotypes was in accordance with the Hardy-Weinberg equilibrium for both cases and controls (Table 2). The distribution of TARC/ CCL17 genotypes between the KD patients and healthy children differed significantly (Table 2). In the comparison of the alleles distribution between the KD patients and the normal control, the TARC/CCL17 SNP rs223895, rs223899, and rs223828 showed significant associations with $\mathrm{KD}$ under three genetic models (genotype model: $P=0.0262, P=0.0095$, and $P=0.0028$; dominant model: $P=0.0149, P=0.0031$, and $P=$ 0.0007; allelic model: $P=0.0065, P=0.0033$, and $P=0.0013$, respectively). Furthermore, the T-allele frequency of rs 223895 , A-allele frequency of rs223899, and T-allele frequency of rs223828 were all significantly lower in patients with KD than that in the normal control (Table 2; $P=0.0062, P=0.0025$, and $P=0.0011$, respectively).

Table 1. Basal characteristics of patients with KD and normal controls

\begin{tabular}{lcc}
\hline Characteristic & $\begin{array}{c}\text { Patients with KD } \\
(n=381)\end{array}$ & $\begin{array}{c}\text { Normal controls } \\
(n=564)\end{array}$ \\
\hline Male gender, $n(\%)$ & $247(66.8)$ & $313(55.5)$ \\
Mean (SD), age (y) & $1.7 \pm 1.6$ & $5.4 \pm 3.7$ \\
Age range (y) & $0-11$ & $0-23$ \\
CAL formation & $37(9.7 \%)$ & \\
Aneurysm formation & $16(4.2 \%)$ & \\
IVIG resistance & $49(12.9 \%)$ & \\
\hline
\end{tabular}

CAL, coronary artery lesion; IVIG, intravenous immunoglobulin; KD, Kawasaki disease.
Association Between TARC/CCL17 Genetic Polymorphism and CAL, Aneurysm Formation, or IVIG Responsiveness

In the comparison of the distribution of alleles and the risk of CAL formation, as shown in Table 3, there were no differences between genotypes of TARC/CCL17 and CAL formation. Further analysis found that KD patients with rs16956811 GG genotype $(P=0.0451)$ and $\mathrm{rs} 223828 \mathrm{CC}$ genotype $(P=$ 0.0389 ) had higher risk of aneurysm formation; however, the significance disappeared after Bonferroni correction (Table 4). Moreover, genetic polymorphisms of rs223895, rs4784805, and rs223828 were also associated with aneurysm formation; however, also in this case, the significance disappeared after Bonferroni correction (see Supplementary Table S1 online). In addition, pharmacogenomics approaches indicated that patients with KD have A allele of rs4784805 $(P=0.0037$ and $P=0.0046$, respectively) with better IVIG treatment response (Table 5).

\section{Plasma TARC/CCL17 Levels in the KD Patients and Controls}

We measured the plasma TARC/CCL17 protein expressions in the KD patients $(n=92)$ and age-matched febrile controls $(n=99)$ using enzyme-linked immunosorbent assay kits. As shown in Figure 1, we found higher TARC/CCL17 levels in the KD patients than that in the controls $(398.01 \pm 35.18$ and $127.33 \pm 14.18 \mathrm{pg} / \mathrm{ml}$, respectively; $P<0.001)$. The TARC/ CCL17 levels were greatly decreased after IVIG treatment, and the lowest levels were noted at the subacute stage at least 3 wk after IVIG treatment in KD patients $(303.94 \pm 29.51$ and $160.14 \pm 14.64 \mathrm{pg} / \mathrm{ml}$, respectively; both $P<0.001)$. In addition, we found that the plasma TARC/CCL17 levels of pre-IVIG, post-IVIG, and subacute stages did not differ significantly in

Table 2. Genotype and allele frequencies of the CCL17gene in controls and patients with KD

\begin{tabular}{|c|c|c|c|c|c|c|c|c|c|c|}
\hline & & Case (\%) & Control (\%) & Allele & Case (\%) & Control (\%) & Genotype & Dominant & Recessive & Allelic \\
\hline & Genotype & $(n=381)$ & $(n=564)$ & & $(n=381)$ & $(n=564)$ & $P$ value & $P$ value & $P$ value & $P$ value \\
\hline \multirow[t]{3}{*}{ rs223895 } & $\mathrm{TT}$ & $50(13.9)$ & $98(18.8)$ & $\mathrm{T}$ & $263(36.5)$ & $448(43.0)$ & $0.0262^{*}$ & $0.0149 *$ & 0.0548 & $0.0065^{* *}$ \\
\hline & $\mathrm{CT}$ & $163(45.3)$ & $252(48.3)$ & C & $457(63.5)$ & $594(57.0)$ & & & & \\
\hline & CC & $147(40.8)$ & $171(32.8)$ & & & & & & & \\
\hline \multirow[t]{3}{*}{ rs4784805 } & $\mathrm{AA}$ & $2(0.6)$ & $3(0.6)$ & A & $42(5.9)$ & $61(5.8)$ & 0.9857 & 0.8688 & 0.9983 & 0.8759 \\
\hline & $A C$ & $38(10.7)$ & $55(10.4)$ & $C$ & $666(94.1)$ & $999(94.2)$ & & & & \\
\hline & CC & $314(88.7)$ & $472(89.0)$ & & & & & & & \\
\hline \multirow[t]{3}{*}{ rs16956811 } & GG & $1(0.3)$ & $0(0.0)$ & G & $11(1.5)$ & $12(1.2)$ & 0.4799 & 0.6709 & 0.2306 & 0.5064 \\
\hline & GT & $9(2.5)$ & $12(2.3)$ & $T$ & 705 (98.5) & $1016(98.8)$ & & & & \\
\hline & $\mathrm{TT}$ & $348(97.2)$ & $502(97.7)$ & & & & & & & \\
\hline \multirow[t]{3}{*}{ rs223899 } & $\mathrm{AA}$ & $32(8.9)$ & $63(12.4)$ & A & $216(30.1)$ & $374(36.9)$ & $0.0095^{* *}$ & $0.0031 * *$ & 0.1032 & $0.0033^{* *}$ \\
\hline & $A G$ & $152(42.3)$ & $248(48.9)$ & G & $502(69.9)$ & $640(63.1)$ & & & & \\
\hline & GG & $175(48.8)$ & $196(38.7)$ & & & & & & & \\
\hline \multirow[t]{3}{*}{ rs223828 } & $\mathrm{TT}$ & $26(7.5)$ & $53(10.2)$ & $T$ & $183(26.2)$ & $348(33.5)$ & $0.0029 * *$ & $0.0007^{* *}$ & 0.1680 & $0.0013^{* *}$ \\
\hline & $\mathrm{TC}$ & $131(37.5)$ & $242(46.5)$ & $C$ & $515(73.8)$ & $692(66.5)$ & & & & \\
\hline & $\mathrm{CC}$ & $192(55.0)$ & $225(43.3)$ & & & & & & & \\
\hline
\end{tabular}

KD, Kawasaki disease.

*Significant $(0.01 \leq P<0.05)$ values are in bold. ${ }^{* *}$ Significant $(P<0.01)$ values are in bold. 
Table 3. Genotype and allele frequencies of CCL17 gene in patients having KD with or without CAL formation

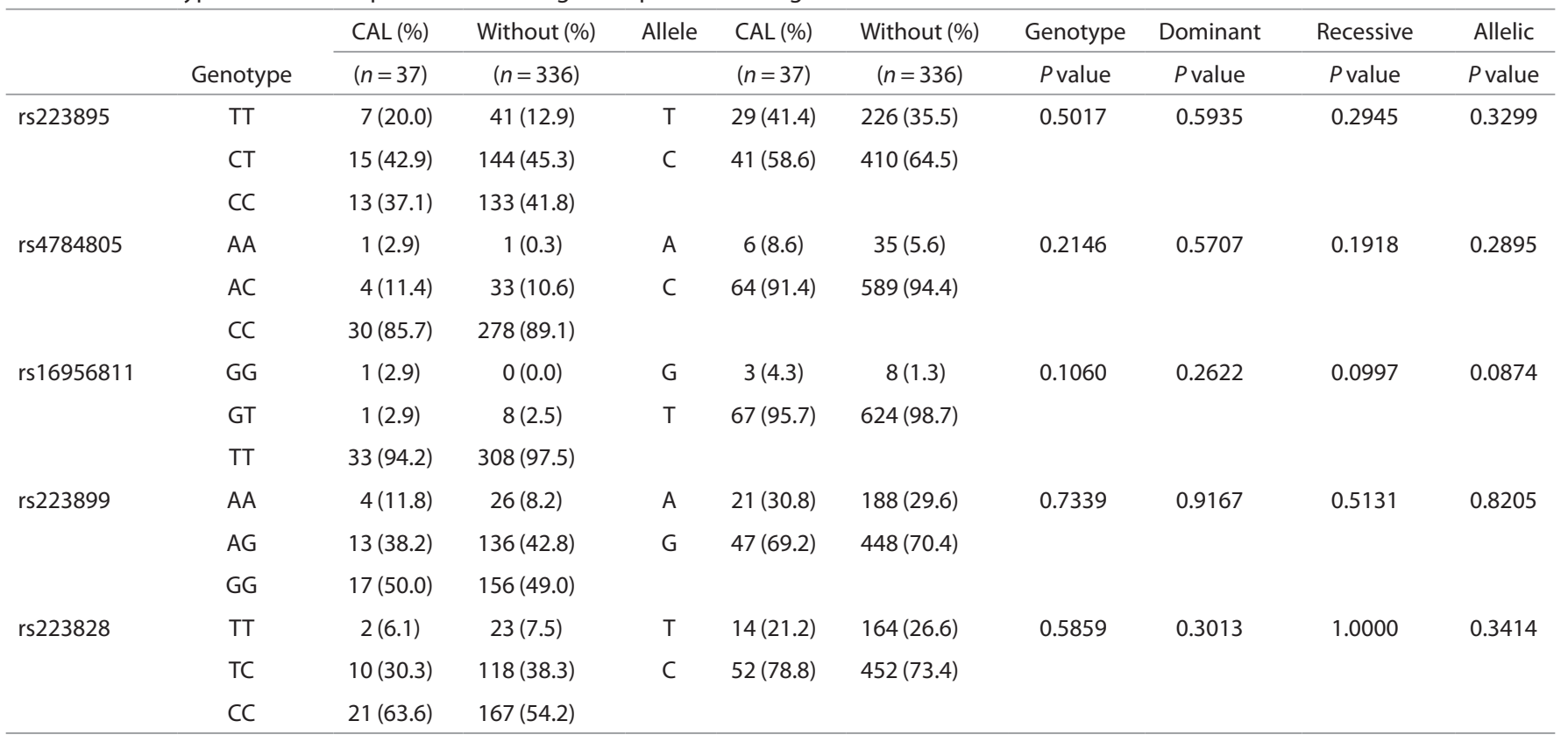

CAL, coronary artery lesion; KD, Kawasaki disease.

Table 4. Genotype and allele frequencies of the CCL17 gene in patients with KD with aneurysm or without aneurysm

\begin{tabular}{|c|c|c|c|c|c|c|c|c|c|c|}
\hline & Genotype & $\begin{array}{l}\text { Aneurysm } \\
(n=16)(\%)\end{array}$ & $\begin{array}{l}\text { Without aneurysm } \\
\qquad(n=362)(\%)\end{array}$ & Allele & $\begin{array}{l}\text { Aneurysm } \\
(n=16)(\%)\end{array}$ & $\begin{array}{l}\text { Without aneurysm } \\
\qquad(n=362)(\%)\end{array}$ & $\begin{array}{l}\text { Genotype } \\
\text { ( } P \text { value })\end{array}$ & $\begin{array}{l}\text { Dominant } \\
(P \text { value })\end{array}$ & $\begin{array}{l}\text { Recessive } \\
(P \text { value })\end{array}$ & $\begin{array}{l}\text { Allelic } \\
\text { (Pvalue) }\end{array}$ \\
\hline \multirow[t]{2}{*}{ rs223895 } & $\mathrm{TT}$ & $2(13.3)$ & $48(14.0)$ & $\mathrm{T}$ & $8(26.7)$ & $252(36.8)$ & 0.2860 & 0.1302 & 1.0000 & 0.2569 \\
\hline & $\mathrm{CC}$ & $9(60.0)$ & $138(40.4)$ & & & & & & & \\
\hline rs4784805 & $A A$ & $0(0.0)$ & $2(0.6)$ & A & $0(0.0)$ & $41(6.1)$ & 0.4478 & 0.2339 & 1.0000 & 0.2489 \\
\hline \multirow[t]{3}{*}{ rs16956811 } & GG & $1(6.2)$ & $0(0.0)$ & G & $2(6.3)$ & $9(1.3)$ & 0.0502 & 0.3733 & $0.0451 *$ & 0.0840 \\
\hline & GT & $0(0.0)$ & $9(2.7)$ & $\mathrm{T}$ & 30 (93.7) & 669 (98.7) & & & & \\
\hline & $\mathrm{TT}$ & $15(93.8)$ & $330(97.3)$ & & & & & & & \\
\hline rs223899 & $A A$ & $2(12.5)$ & $30(8.8)$ & A & $8(25.0)$ & $206(30.3)$ & 0.3618 & 0.2646 & 0.6453 & 0.5233 \\
\hline \multirow{2}{*}{ rs223828 } & $\mathrm{TC}$ & $2(12.5)$ & $127(38.5)$ & $C$ & $28(87.5)$ & $483(73.2)$ & & & & \\
\hline & $\mathrm{CC}$ & $13(81.2)$ & $178(53.9)$ & & & & & & & \\
\hline
\end{tabular}

KD, Kawasaki disease.

*Significant $(0.01 \leq P<0.05)$ values are in bold.

the occurrence of CAL, CAA, and the responsiveness in $\mathrm{KD}$ patients (data not shown; all $P>0.1$ ).

Plasma TARC/CCL17 Levels in the Different Genotypes of KD Patients and Controls

We explored the functional effect of the genetic polymorphisms of TARC gene expression. As shown in Table 6, TARC/ CCL17 levels were higher in the patients with KD than that in the controls $(P<0.001)$. However, no significant correlation between genetic polymorphisms and protein expression was noticed.

\section{DISCUSSION}

The immunopathogenesis of KD remains unknown. Several studies have pointed out an imbalanced immunity between Th1 and Th2 reaction in patients with $\mathrm{KD}$. Sekiya et al. (16) first demonstrated a functional SNP in the $5^{\prime}$-flanking region $(-431 \mathrm{C} / \mathrm{T})$ of the TARC/CCL17 gene; however, there was no significant 


\section{Articles | Lee et al.}

Table 5. Genotype and allele frequencies of the CCL17 gene in patients with KD responding or not responding to IVIG treatment

\begin{tabular}{|c|c|c|c|c|c|c|c|c|c|c|}
\hline & Genotype & $\begin{array}{l}\text { Resistant } \\
(n=49)(\%)\end{array}$ & $\begin{array}{l}\text { Responsive } \\
(n=332)(\%)\end{array}$ & Allele & $\begin{array}{c}\text { Resistant } \\
(n=49)(\%)\end{array}$ & $\begin{array}{l}\text { Responsive } \\
(n=332)(\%)\end{array}$ & $\begin{array}{l}\text { Genotype } \\
(P \text { value })\end{array}$ & $\begin{array}{l}\text { Dominant } \\
(P \text { value })\end{array}$ & $\begin{array}{c}\text { Recessive } \\
(P \text { value })\end{array}$ & $\begin{array}{c}\text { Allelic } \\
\text { (Pvalue) }\end{array}$ \\
\hline \multirow[t]{2}{*}{ rs223895 } & $\mathrm{CC}$ & $4(8.7)$ & $46(14.9)$ & $C$ & $30(32.6)$ & $229(37.2)$ & 0.5261 & 0.7097 & 0.3637 & 0.3963 \\
\hline & $\mathrm{TT}$ & $20(43.5)$ & $125(40.6)$ & & & & & & & \\
\hline rs4784805 & AA & $0(0.0)$ & $2(0.7)$ & A & $0(0.0)$ & $41(6.8)$ & $0.0200 *$ & $0.0046 * *$ & 1.0000 & $0.0037^{* *}$ \\
\hline \multirow[t]{3}{*}{ rs16956811 } & GG & $1(2.2)$ & $0(0.0)$ & G & $4(4.3)$ & $7(1.1)$ & 0.0597 & 0.1296 & 0.1307 & $0.0436 *$ \\
\hline & GT & $2(4.3)$ & $7(2.3)$ & $\mathrm{T}$ & 88 (95.7) & 605 (98.9) & & & & \\
\hline & $\mathrm{TT}$ & $43(93.5)$ & 299 (97.7) & & & & & & & \\
\hline rs223899 & $\mathrm{AA}$ & $2(4.5)$ & $30(9.7)$ & A & $24(26.7)$ & $189(30.7)$ & 0.5127 & 0.7317 & 0.4020 & 0.4382 \\
\hline \multirow{2}{*}{ rs223828 } & TC & $13(30.9)$ & $115(38.2)$ & $C$ & $67(79.8)$ & 439 (72.9) & & & & \\
\hline & $\mathrm{CC}$ & $27(64.3)$ & $162(53.8)$ & & & & & & & \\
\hline
\end{tabular}

IVIG, intravenous immunoglobulin

*Significant $(0.01 \leq P<0.05)$ values are in bold. **Significant $(P<0.01)$ values are in bold.

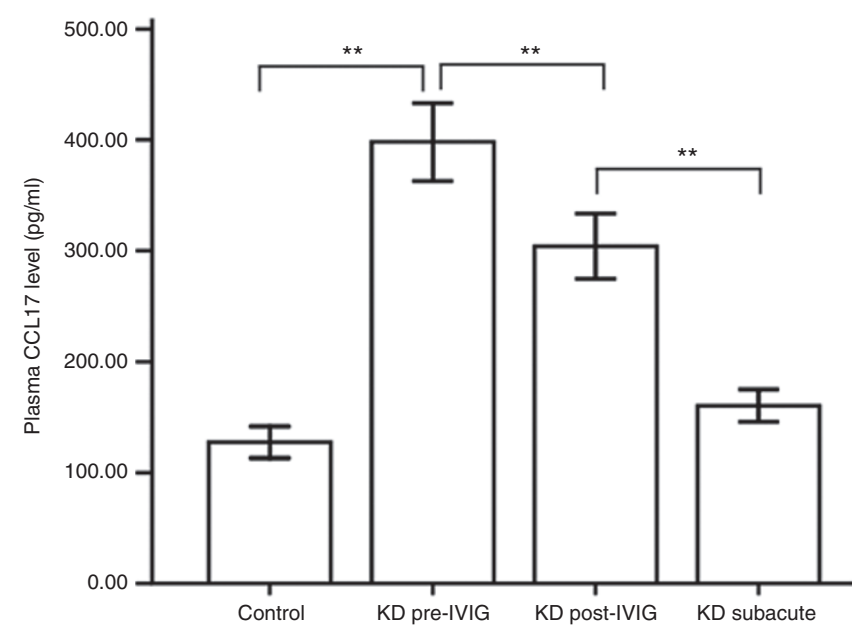

Figure 1. Comparison of plasma TARC/CCL17 levels by enzyme-linked immunosorbent assay (ELISA) between controls $(n=99)$ and patients with Kawasaki disease (KD) $(n=92)$. There were higher TARC/CCL17 levels in the KD patients than in the controls before intravenous immunoglobulin (IVIG) treatment. Furthermore, the TARC/CCL17 levels were greatly decreased after IVIG treatment and the lowest levels at subacute stage at least 3 wk after IVIG treatment in KD patients. ${ }^{* *} P<0.01$. Data are presented as mean $\pm \mathrm{SE}$.

association of the SNP with susceptibility to bronchial asthma and atopic dermatitis $(10,16)$. In this study, we report the correlation between TARC/CCL17 genetic polymorphisms and KD. Furthermore, we also found that plasma TARC/CCL17 protein levels changed dynamically over the course of the illness. Plasma TARC/CCL17 levels were higher in KD patients than that in controls $(P<0.001)$ before IVIG treatment. After IVIG treatment, plasma TARC/CCL17 levels decreased significantly $(P<0.001)$. However, the change of TARC/CCL17 levels was not associated with IVIG treatment response and the occurrence of CAL and CAA. The plasma TARC/CCL17 levels were markedly increased in patients with $\mathrm{KD}$ in the acute stage and greatly decreased after IVIG treatment.

TARC/CCL17, a ligand for CC chemokine receptor 4 and CC chemokine receptor 8 , serves for the recruitment and migration of lymphocytes of the Th2 phenotype $(8,9)$ and direct T-cell and macrophage recruitment into areas of allergic inflammation. In human and animal studies, it is demonstrated that TARC/CCL17 mRNA and protein are potently induced by the Th2 cytokine, interleukin-4 (IL-4), through induction of promoter binding of signal transducer and activator of transcription 6 (STAT6) $(17,18)$. IL-4/signal transducer and activator of transcription 6 signaling during T-cell development contributes to polarized patterns of cytokine expression manifested by differentiated Th cells (19). IL-4, mainly produced by Th2 $\mathrm{T}$ cells, mast cells, basophils, and eosinophils, appears to be a crucial factor in allergic responses (20). However, prolonged IL-4 stimulation might result in the downregulation of TARC/CCL17 expression through classical negative-feedback loops including the activation of suppressors of cytokine signaling families (21). Hijnen et al. (22) demonstrated that TARC/CCL17 levels in patients with atopic dermatitis were significantly higher than those in healthy controls. Furthermore, they can be objective parameters for disease severity-specific atopic dermatitis and treatment monitoring. In our KD patients, we found that significantly high levels of TARC/CCL17 have also been observed than those in the controls, which was compatible with our previous findings of increased IL-4/IL-5 expression in patients with KD (13). Moreover, our previous study showed that IL-4, IL-5, and eotaxin increased significantly after IVIG treatment (13). Not 
Table 6. Differences in TARC levels among patients with KD and control subjects stratified by CCL17 genotype

\begin{tabular}{|c|c|c|c|c|c|}
\hline \multirow[b]{2}{*}{ SNP } & \multirow[b]{2}{*}{ Genotype } & \multicolumn{2}{|c|}{ Patients with KD } & \multicolumn{2}{|c|}{ Control subjects } \\
\hline & & $n$ & Mean \pm SD & $n$ & Mean \pm SD \\
\hline \multirow[t]{4}{*}{ rs223895 } & TT & 9 & $303.27 \pm 233.91^{\mathrm{a}}$ & 14 & $91.98 \pm 69.23$ \\
\hline & $\mathrm{CT}$ & 39 & $465.39 \pm 387.14$ & 50 & $121.94 \pm 108.90$ \\
\hline & CC & 30 & $296.22 \pm 244.63$ & 27 & $108.91 \pm 108.85$ \\
\hline & $P$ value & & 0.0802 & & 0.6138 \\
\hline \multirow[t]{4}{*}{ rs4784805 } & $A A$ & 0 & - & 0 & - \\
\hline & $A C$ & 7 & $317.44 \pm 184.21$ & 13 & $113.40 \pm 82.09$ \\
\hline & $\mathrm{CC}$ & 71 & $386.70 \pm 341.92$ & 80 & $117.24 \pm 108.74$ \\
\hline & $P$ value & & 0.6002 & & 0.9036 \\
\hline \multirow[t]{4}{*}{ rs16956811 } & GG & 0 & - & 0 & - \\
\hline & GT & 1 & 506.37 & 1 & 151.03 \\
\hline & TT & 77 & $386.51 \pm 332.97$ & 93 & $111.83 \pm 104.53$ \\
\hline & $P$ value & & 0.7216 & & 0.7100 \\
\hline \multirow[t]{4}{*}{ rs223899 } & $A A$ & 7 & $327.44 \pm 236.43$ & 8 & $95.73 \pm 70.67$ \\
\hline & AG & 37 & $458.14 \pm 384.14$ & 47 & $120.20 \pm 113.62$ \\
\hline & GG & 34 & $300.06 \pm 236.13$ & 34 & $110.07 \pm 99.02$ \\
\hline & $P$ value & & 0.1059 & & 0.7982 \\
\hline \multirow[t]{4}{*}{ rs223828 } & TT & 3 & $196.16 \pm 27.23$ & 5 & $49.74 \pm 19.49$ \\
\hline & TC & 34 & $485.80 \pm 394.44$ & 47 & $111.26 \pm 91.24$ \\
\hline & CC & 38 & $310.38 \pm 262.43$ & 42 & $120.47 \pm 119.59$ \\
\hline & $P$ value & & 0.0506 & & 0.3538 \\
\hline
\end{tabular}

KD, Kawasaki disease; SNP, single-nucleotide polymorphism.

${ }^{a}$ Data represent means \pm SD.

surprisingly, the TARC/CCL17 levels were greatly decreased after IVIG treatment in KD patients, which raises the possibility of the activation of negative-feedback loops $(23,24)$. Of note, there were still higher TARC/CCL17 levels at a subacute stage than those in the controls $(P<0.001)$. However, we found that the plasma TARC/CCL17 levels of pre-IVIG, post-IVIG, and subacute stages did not differ significantly in the occurrence of CAL, CAA, and the responsiveness in the KD patients. However, high levels of TARC/CCL17 mRNA expression are seen in some, but not all, human arteries with advanced atherosclerotic lesions which could play a role in mononuclear cell recruitment into atherosclerotic lesions and influence the subsequent inflammatory response (25).

In consonance with the findings of Sekiya et al. (16) and Tsunemi et al. (22) in allergic disease, we also found increased plasma TARC/CCL17 levels in the KD patients than those in the controls, but the differences were not significant. Indeed, we found that the TARC/CCL17 levels were not associated with the genotypes of TARC/CCL17 in the patients with $\mathrm{KD}$ and in the controls, which reflects that the upregulation of TARC/CCL17 levels might be due to the disease itself and not because of the genotype difference.

Genetics might play an important role in the pathogenesis of $\mathrm{KD}$ (26). Genome-wide association study for KD was firstly performed by Burgner et al. (27). A total of 40 SNPs and 6 haplotypes were confirmed in an independent cohort of KD families. In Asian population, Onouchi et al. (28) identified the susceptibility loci on chromosome 19q13.2 and further confirmed the functional SNP of inositol 1, 4, 5-trisphosphate 3-kinase C in the disease activity of KD. Recently, it has been demonstrated that rs28493229 is associated with susceptibility to $\mathrm{KD}$ and CAL formation $(28,29)$. We have also demonstrated that the A allele of rs72689236 located in the $5^{\prime}$-untranslated region of caspase 3 is very likely to be a risk allele in the development of aneurysm in patients with $\mathrm{KD}(30)$ and the association of CTLA-4 (+49) $\mathrm{A} / \mathrm{G}$ polymorphism with the CAL formation of $\mathrm{KD}$, particularly in female patients (31). In this study, we revealed that TARC/ CCL17 gene polymorphisms have an association with the susceptibility of KD and were significantly associated with disease outcomes of KD patients in a Taiwanese population. Indeed, to the best of our knowledge, the allelic distribution of TARC/ CCL17 between ethnic groups is still unknown. Future studies of TARC/CCL17 genotypes in patients with $\mathrm{KD}$ of other ancestries could prove to be quite interesting.

This study has potential limitations that should be reviewed. First, there were still higher TARC/CCL17 levels at the subacute stage at least $3 \mathrm{wk}$ after IVIG treatment in KD patients as compared with the controls, and this should be ascertained through longitudinal studies to elucidate the time-dependent changes in TARC/CCL17 levels, especially during the convalescent stage of $\mathrm{KD}$. Second, we cannot exclude the possibility that the higher TARC/CCL17 levels in the KD patients as compared with the controls were due to a longer duration of fever in the KD patients than that in the controls. Third, to identify low-frequency genetic polymorphisms in TARC/CCL17, application of direct sequencing is needed.

\section{Conclusion}

This study provides the first evidence supporting the association of TARC/CCL17 polymorphisms with the susceptibility to $\mathrm{KD}$ and clinical outcome as well as significantly increased TARC/CCL17 levels in KD patients. The role of elevated TARC levels in KD patients remains unknown. Further studies are warranted to investigate the pathophysiological basis for these findings with regard to $\mathrm{KD}$.

\section{METHODS}

\section{Patients Studied}

A total of 381 patients with KD and 564 controls were enrolled in this study. The prevalence of KD is $<1$ in 1,000 children in a Taiwanese population. Therefore, we assumed that there was no KD case in the control group. Blood samples from the febrile age-matched control patients, who were admitted for upper and/or lower respiratory tract infections (including acute bronchiolitis, acute pharyngitis, acute bronchitis, croup, and acute tonsillitis), were used for cytokine comparison. All KD patients were initially treated with a single dose of IVIG $(2 \mathrm{~g} / \mathrm{kg})$ during a $12-\mathrm{h}$ period. This study was approved by the Institutional Review Board of the Chang Gung Memorial Hospital, and informed consent was obtained from either the parents or guardians of the children. Blood samples were collected within $24 \mathrm{~h}$ before IVIG treatment (pre-IVIG stage), within $3 \mathrm{~d}$ after IVIG treatment (post-IVIG stage), and at least 3 wk after IVIG treatment (subacute stage). Patients whose symptoms did not fit the KD criteria or those who had suffered from an acute fever for $<5 \mathrm{~d}$ were excluded. All 
the KD patients underwent two-dimensional pulse Doppler and color flow imaging at least three times within $8 \mathrm{wk}$ from the onset of the illness. If patients had abnormal coronary arteries, echocardiographic follow-up was scheduled every 3-6 mo within the first year, and then once annually until the affected coronary arteries resumed being normal as our previous reports $(32,33)$. Echocardiography was performed with a SONOS 5500 or 7500 cardiac scanner (Philips, Andover, MA). Five- to eight- $\mathrm{MHz}$ sector phased array transducers were used for this study. Two-dimensional echocardiography was performed to visualize the diameter of the right and left coronary arteries on the parasternal short-axis view of the aorta (4). According to the Japanese Ministry of Health guidelines, a CAL was defined by the internal diameter of the coronary artery being $>3 \mathrm{~mm}(4 \mathrm{~mm}$, if the subject was older than $5 \mathrm{y}$ of age) or the internal diameter of a segment being at least 1.5 times that of an adjacent segment, as observed in the echocardiogram. KD patients with coronary artery ectasia or dilation that was disappearing within the initial $8 \mathrm{wk}$ after the onset of illness were defined as transient ectasia and not judged as CAL $(30,34)$. In addition, coronary arteries were classified on the basis of presence or absence of aneurysms according to the criteria from the Japanese Circulation Society Joint Working Group. A CAA (including medium and giant aneurysms) was defined by the internal diameter of the coronary artery being at least $4 \mathrm{~mm}$ or, in children older than $5 \mathrm{y}$ of age, the internal diameter of a segment being at least 1.5 times that of an adjacent segment as observed from echocardiography $(30,34)$. IVIG responsiveness was defined as defervescence $48 \mathrm{~h}$ after the completion of IVIG treatment and no fever (defined as a temperature $>38^{\circ} \mathrm{C}$ ) recurrence for at least $7 \mathrm{~d}$ after IVIG, with marked improvement or normalization of inflammatory signs $(33,35)$. Blood samples were immediately placed in tubes containing heparin, and the remaining aliquots of plasma were stored at $-80^{\circ} \mathrm{C}$ until assay.

\section{DNA Extraction}

Blood cells were subjected to DNA extraction by treating them first with cell lysis solution (Cat. No.:158908; Qiagen Sciences, Germantown, MD) and RNase A (Cat. No.: R6513, Sigma-Aldrich, St. Louis, MO) for digestion ribonuclease for $15 \mathrm{~min}$ at $37^{\circ} \mathrm{C}$, and then protein precipitation solution (Cat. No.:158912, Qiagen Sciences) for digestion of nuclear protein. Total DNA was harvested by using Puregen manufacturer's followed by isopropanol precipitation.

\section{Genotyping}

Five tagging SNPs of TARC/CCL17 (rs223895, rs4784805, rs16956811, rs223899, and rs223828) with a minimum allele frequency of $>1 \%$ in the Han Chinese in Beijing population were selected from the HapMap database (http://hapmap.ncbi.nlm.nih.gov/). All polymorphisms of TARC/CCL17 located in the intron. Genomic DNA was extracted from whole-blood samples by using the standard method as described in our previous study (29). Genotyping was carried out using the TaqMan Allelic Discrimination Assay (Applied Biosystems, Foster City, CA). Briefly, the PCR was performed by using a 96-well microplate with the ABI 7500 Realtime PCR system (Applied Biosystems). The thermal cycle conditions were as follows: denaturing at $95^{\circ} \mathrm{C}$ for $10 \mathrm{~min}$, followed by 40 cycles of denaturing at $92^{\circ} \mathrm{C}$ for $15 \mathrm{~s}$, and annealing and extension at $60^{\circ} \mathrm{C}$ for $1 \mathrm{~min}$. After the PCR, fluorescence was measured and analyzed using the System SDS software version 1.3.1 (Applied Biosystems). The average genotyping successful rate in our laboratory is $\sim 95.7 \%$, so some individuals are without genotype data.

\section{Measurement of Cytokines by Enzyme-Linked Immunoassay} We used enzyme-linked immunoassays to measure TARC/CCL17 (human TARC/CCL17; R\&D Systems, Minneapolis, MN) according to the manufacturers' instructions.

\section{Statistical Analysis}

All data are presented as mean \pm SE. Quantitative data were analyzed by using the Student's $t$-test or one-way ANOVA when appropriate. The least significant difference test was used for post hoc testing where appropriate. Changes in the data before and after IVIG treatment as well as subacute stage were tested by the paired sample $t$-test. The genotypes and allele frequencies associated with the KD patients and disease outcomes were tested by $\chi^{2}$ test or Fisher's exact test when appreciate. Two-sided $P$ values $<0.05$ were considered statistically significant. All statistical tests were performed using SPSS version 13.0 for Windows XP (SPSS, Chicago, IL).

\section{SUPPLEMENTARY MATERIAL}

Supplementary material is linked to the online version of the paper at http:// www.nature.com/pr

\section{STATEMENT OF FINANCIAL SUPPORT}

This study was supported by grants from the Taiwan National Science Council (NSC 99-2314-B-182A-032-MY2 and NSC 100-2314-B-182-061-MY3) and grants from Chang Gung Memorial Hospital (CMRPG8A021 and CMRPG8A0481)

Disclosure: The authors have indicated they have no financial relationships relevant to this article to disclose.

\section{REFERENCES}

1. Kawasaki T, Kosaki F, Okawa S, Shigematsu I, Yanagawa H. A new infantile acute febrile mucocutaneous lymph node syndrome (MLNS) prevailing in Japan. Pediatrics 1974;54:271-6.

2. Wang CL, Wu YT, Liu CA, Kuo HC, Yang KD. Kawasaki disease: infection, immunity and genetics. Pediatr Infect Dis J 2005;24:998-1004.

3. Burns JC, Glodé MP. Kawasaki syndrome. Lancet 2004;364:533-44.

4. Liang CD, Kuo HC, Yang KD, Wang CL, Ko SF. Coronary artery fistula associated with Kawasaki disease. Am Heart J 2009;157:584-8.

5. Newburger JW, Takahashi M, Gerber MA, et al.; Committee on Rheumatic Fever, Endocarditis, and Kawasaki Disease, Council on Cardiovascular Disease in the Young, American Heart Association. Diagnosis, treatment, and long-term management of Kawasaki disease: a statement for health professionals from the Committee on Rheumatic Fever, Endocarditis, and Kawasaki Disease, Council on Cardiovascular Disease in the Young, American Heart Association. Pediatrics 2004;114:1708-33.

6. Taubert KA, Rowley AH, Shulman ST. Nationwide survey of Kawasaki disease and acute rheumatic fever. J Pediatr 1991;119:279-82.

7. Imai T, Yoshida T, Baba M, Nishimura M, Kakizaki M, Yoshie O. Molecular cloning of a novel T cell-directed CC chemokine expressed in thymus by signal sequence trap using Epstein-Barr virus vector. J Biol Chem 1996;271:21514-21.

8. Imai T, Baba M, Nishimura M, Kakizaki M, Takagi S, Yoshie O. The T celldirected CC chemokine TARC is a highly specific biological ligand for CC chemokine receptor 4. J Biol Chem 1997;272:15036-42.

9. Imai T, Nagira M, Takagi S, et al. Selective recruitment of CCR4-bearing Th2 cells toward antigen-presenting cells by the CC chemokines thymus and activation-regulated chemokine and macrophage-derived chemokine. Int Immunol 1999;11:81-8.

10. Tsunemi $\mathrm{Y}$, Komine M, Sekiya $\mathrm{T}$, et al. The $-431 \mathrm{C}>\mathrm{T}$ polymorphism of thymus and activation-regulated chemokine increases the promoter activity but is not associated with susceptibility to atopic dermatitis in Japanese patients. Exp Dermatol 2004;13:715-9.

11. Kakinuma T, Nakamura K, Wakugawa M, et al. Thymus and activationregulated chemokine in atopic dermatitis: serum thymus and activationregulated chemokine level is closely related with disease activity. J Allergy Clin Immunol 2001;107:535-41.

12. Sekiya T, Yamada H, Yamaguchi M, et al. Increased levels of a TH2-type CC chemokine thymus and activation-regulated chemokine (TARC) in serum and induced sputum of asthmatics. Allergy 2002;57:173-7.

13. Kuo HC, Wang CL, Liang CD, et al. Association of lower eosinophil-related T helper 2 (Th2) cytokines with coronary artery lesions in Kawasaki disease. Pediatr Allergy Immunol 2009;20:266-72.

14. Brosius CL, Newburger JW, Burns JC, Hojnowski-Diaz P, Zierler S, Leung DY. Increased prevalence of atopic dermatitis in Kawasaki disease. Pediatr Infect Dis J 1988;7:863-6.

15. Webster RJ, Carter KW, Warrington NM, et al. Hospitalisation with infection, asthma and allergy in Kawasaki disease patients and their families: genealogical analysis using linked population data. PLoS ONE 2011;6:e28004. 
16. Sekiya T, Tsunemi Y, Miyamasu M, et al. Variations in the human Th2specific chemokine TARC gene. Immunogenetics 2003;54:742-5.

17. Liddiard K, Welch JS, Lozach J, Heinz S, Glass CK, Greaves DR. Interleukin-4 induction of the CC chemokine TARC (CCL17) in murine macrophages is mediated by multiple STAT6 sites in the TARC gene promoter. BMC Mol Biol 2006;7:45.

18. Wirnsberger G, Hebenstreit D, Posselt G, Horejs-Hoeck J, Duschl A. IL-4 induces expression of TARC/CCL17 via two STAT6 binding sites. Eur J Immunol 2006;36:1882-91.

19. Egwuagu CE, Yu CR, Zhang M, Mahdi RM, Kim SJ, Gery I. Suppressors of cytokine signaling proteins are differentially expressed in Th1 and Th2 cells: implications for Th cell lineage commitment and maintenance. J Immunol 2002;168:3181-7.

20. Nelms K, Keegan AD, Zamorano J, Ryan JJ, Paul WE. The IL-4 receptor: signaling mechanisms and biologic functions. Annu Rev Immunol 1999;17:701-38.

21. Kubo M, Hanada T, Yoshimura A. Suppressors of cytokine signaling and immunity. Nat Immunol 2003;4:1169-76.

22. Hijnen D, De Bruin-Weller M, Oosting B, et al. Serum thymus and activation-regulated chemokine (TARC) and cutaneous $\mathrm{T}$ cell- attracting chemokine (CTACK) levels in allergic diseases: TARC and CTACK are disease-specific markers for atopic dermatitis. J Allergy Clin Immunol 2004;113:334-40.

23. Wang GB, Li CR, Zu Y. [Change of MyD88-independent signal transduction of Toll-like receptor 4 in immunological pathogenesis of Kawasaki disease]. Zhonghua Er Ke Za Zhi 2007;45:818-23.

24. Ogata S, Ogihara Y, Nomoto K, et al. Clinical score and transcript abundance patterns identify Kawasaki disease patients who may benefit from addition of methylprednisolone. Pediatr Res 2009;66:577-84.

25. Greaves DR, Häkkinen T, Lucas AD, et al. Linked chromosome 16q13 chemokines, macrophage-derived chemokine, fractalkine, and thymus- and activation-regulated chemokine, are expressed in human atherosclerotic lesions. Arterioscler Thromb Vasc Biol 2001;21:923-9.

26. Kuo HC, Chang WC. Genetic polymorphisms in Kawasaki disease. Acta Pharmacol Sin 2011;32:1193-8.

27. Burgner D, Davila S, Breunis WB, et al.; International Kawasaki Disease Genetics Consortium. A genome-wide association study identifies novel and functionally related susceptibility Loci for Kawasaki disease. PLoS Genet 2009;5:e1000319.

28. Onouchi Y, Gunji T, Burns JC, et al. ITPKC functional polymorphism associated with Kawasaki disease susceptibility and formation of coronary artery aneurysms. Nat Genet 2008;40:35-42.

29. Kuo HC, Yang KD, Juo SH, et al. ITPKC single nucleotide polymorphism associated with the Kawasaki disease in a Taiwanese population. PLoS ONE 2011;6:e17370.

30. Kuo HC, Yu HR, Juo SH, et al. CASP3 gene single-nucleotide polymorphism (rs72689236) and Kawasaki disease in Taiwanese children. J Hum Genet 2011;56:161-5.

31. Kuo HC, Liang CD, Yu HR, et al. CTLA-4, position 49 A/G polymorphism associated with coronary artery lesions in Kawasaki disease. J Clin Immunol 2011;31:240-4.

32. Kuo HC, Onouchi Y, Hsu YW, et al. Polymorphisms of transforming growth factor- $\beta$ signaling pathway and Kawasaki disease in the Taiwanese population. J Hum Genet 2011;56:840-5.

33. Kuo HC, Liang CD, Wang CL, Yu HR, Hwang KP, Yang KD. Serum albumin level predicts initial intravenous immunoglobulin treatment failure in Kawasaki disease. Acta Paediatr 2010;99:1578-83.

34. Shulman ST, De Inocencio J, Hirsch R. Kawasaki disease. Pediatr Clin North Am 1995;42:1205-22.

35. Kuo HC, Yang KD, Liang CD, et al. The relationship of eosinophilia to intravenous immunoglobulin treatment failure in Kawasaki disease. Pediatr Allergy Immunol 2007;18:354-9. 